\title{
3 MARCO TEÓRICO, HIPÓTESIS, OBJETIVOS Y METODOLOGÍA DE LA INVESTIGACIÓN EMPÍRICA
}

El estudio del SI ha de integrarse en un marco teórico general explicativo del entramado de relaciones entre los distintos actores de la infraestructura del sistema presente dentro de los enfoques actuales.

Por esta razón es necesario ahora, describir cual sería el marco teórico que permitiría incorporar el estudio del SI-SSPC de forma que éste pueda aportar elementos enriquecedores al estudio de los SNI.

El presente trabajo no puede vincularse de manera exclusiva con una determinada teoría. La conceptualización del objeto de estudio se ha hecho a partir de aportaciones de diferentes perspectivas teóricas ${ }^{1}$.

De las diferentes perspectivas utilizadas, el trabajo participa en primer lugar, de las aportaciones hechas por Lundvall [1992, pp. 1-19] de que «un sistema nacional de innovación está constituido por elementos y relaciones que interactúan en el marco de la producción, de la difusión y de la utilización de conocimientos nuevos y económicamente útiles..., un sistema nacional de innovación comprende elementos y relaciones circunscriptas a las fronteras de un Estado»....en todo momento, en todas las partes de la economía, nosotros esperamos encontrar procesos continuos de aprendizaje, investigando y descubriendo, produciendo los nuevos productos, las nuevas técnicas, las nuevas formas de organización y los nuevos mercados».

También, y por otro lado, las aportaciones hechas por Bell y Callon [1994, pp. 64-126] definen que «la investigación técnico—económica se basa en una

1 Este método científico se ajustaría a los empleados en la investigación en el campo de la Dirección de Empresas donde «no existe paradigma que domine la lógica de la disciplina ni deseo de buscar un paradigma que restrinja la visión del campo. Modelos y teorías se utilizan como herramientas siempre y cuando nos ayuden a conceptualizar un tema determinado», [Gómez 1996, p. 1]. 
infraestructura y está compuesta por características fundamentales, que son: La movilidad y la circulación de la información, independientemente de los soportes que se utilicen y, definiendo un espacio comprendido por todos los actores que crean un circulo que pasa la información necesaria a la realización de su finalidad de manera inteligible».

Por último, la aportación de Fernández y Conesa [1996, p. 48] que hacen un primer estudio del Sistema de Innovación Español y que definen «el sistema nacional de innovación como el conjunto de elementos y estructuras, que asumen funciones específicas en el proceso de producción, transmisión y almacenamiento de conocimientos y por su capacidad de interacción, mediante la articulación de sus elementos, para la producción de conocimientos y la difusión y utilización de los mismos».

Nuestra propuesta de marco teórico tiene como punto de referencia los modelos estudiados. En el que, reconociendo el desigual valor de las diferentes aportaciones al estudio del SI, se propone un modelo teórico integrado en todo aquello que cada teoría explicativa pueda tener de interés.

En nuestro caso el modelo estará basado en las teorías que se han descrito como dominantes en el pensamiento de los SNI en la actualidad.

En este modelo que proponemos combina el tipo de construcción teórica para explicar «actor» y «estructura». 
FIGURA 3.1. Representación actual del sistema de innovación en el sector sanitario catalán.

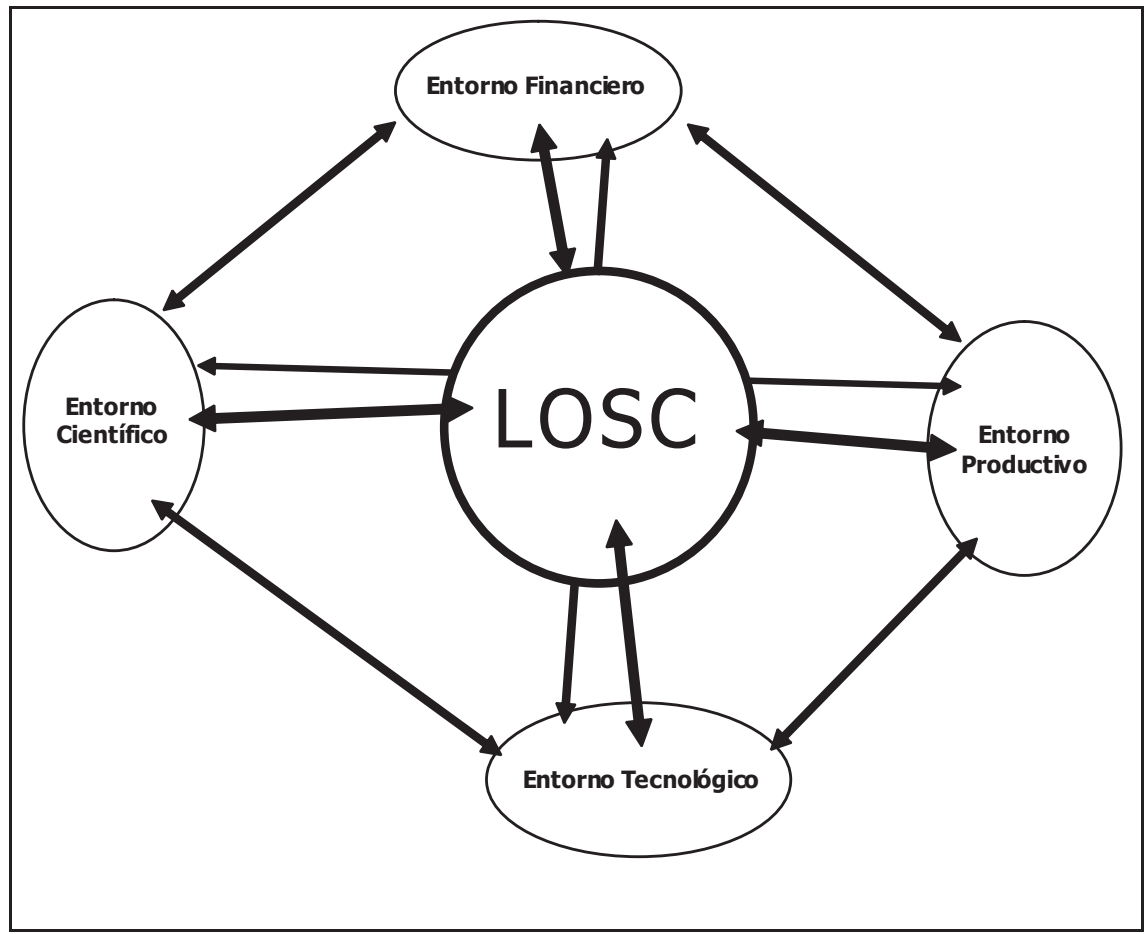

Fuente: Elaboración propia.

Para describir el modelo podemos decir que a partir de la LOSC — párrafo $2^{\circ}$ se crea una «estructura» que tiene como objetivo la ordenación del sistema sanitario público de Catalunya. Además, también se crean los «actores» —artículos 3, 4, 5, 6, 7, 8, 21, 33, 41, 43, 62, 69, 70 - que forman parte de esta estructura. Estos actores asumen funciones específicas en el proceso de producción de salud, producción, transmisión y almacenamiento de conocimientos, y se caracterizan por su capacidad de interacción dentro del SSPC, para la difusión y utilización de esos conocimientos.

\subsection{RECURSOS COMPARTIDOS EN EL SI}

El SI tal como ha sido definido en el capítulo 2 a partir de la integración de las diferentes perspectivas estudiadas, presenta ciertas peculiaridades con relación a los actores.

Los diversos estudios sobre el SI coinciden en reconocer la existencia de un conjunto de externalidades existentes entre «actor» y «estructura». Estas 
externalidades pueden ser identificadas como recursos compartidos por los actores que pertenecen al SI. Recursos que tienen un carácter colectivo para los actores del sistema y privado respecto a los actores del exterior.

La base constitutiva de estos recursos compartidos son la información y los conocimientos que circulan en el interior del sistema productivo. Los recursos compartidos se concretan en un conjunto de recursos propiamente dichos y sobre todo, en una serie de modelos o patrones de relaciones y comportamientos que pueden entenderse como rutinas organizativas que superan el marco del actor individual, se insertan en el conjunto del sistema y permiten la activación de sus potencialidades. La naturaleza de estos recursos es intangible, son difíciles de identificar y son el resultado de una experiencia común de todos los actores.

Los recursos compartidos son factores competitivos interrelacionados siendo unos efectos de otros. Pueden ser identificados para facilitar su comprensión mostrando las diferentes dimensiones que presentan y que son los siguientes ${ }^{2}$.

- La disponibilidad de información.

- Los conocimientos acumulados.

- La gestión de la innovación.

- La red cooperativa

- La flexibilidad.

\subsubsection{LA DISPONIBILIDAD DE INFORMACIÓN}

Dos serían las fuentes de la información que circulan dentro del sistema. Por un lado, la derivada de la producción conjunta de bienes no normalizados. La vía de acceso a esta información es básicamente informal. La proximidad y familiaridad de los actores hace que se den vínculos más allá de las relaciones puramente productivas.

Todo esto fomenta un intercambio continuo de información que permite a los actores y sus directivos la disposición de una base de datos completa y actualizada sobre los diversos aspectos de la producción y de los mercados. Tanto los clientes como los proveedores como los competidores intercambian información.

Por otro lado, está la información suministrada por las instituciones que ofrecen estos servicios. Tanto desde las instituciones públicas como desde las privadas se

2 Este enfoque conceptual de recursos compartidos fue propuesto por Molina [1997, pp. 136141], también llamados recursos generadores de éxito, factores de éxito o factores de dinamización. 
ofrece información de muy diversa índole sobre especificaciones técnicas de procesos, productos y mercados, normativas legales, etc. Esta información es accesible a la totalidad de los miembros del sistema y constituye también una base de datos compartida a la que pueden acudir todos los actores [Brusco, 1993, pp. 235-258].

\subsubsection{LOS CONOCIMIENTOS ACUMULADOS}

Los conocimientos acumulados hacen referencia a toda la experiencia previa acumulada por las personas e instituciones que pertenecen al sistema. La existencia de un «pool» de directivos, médicos, doctores, enfermeros, técnicos y operarios cualificados con conocimientos específicos, además de la experiencia que las instituciones aportan al sistema proporciona una riqueza y diversidad importante en la consolidación del modelo sanitario catalán.

Para los empleados, la inversión en estos conocimientos viene justificada por la seguridad que da el mercado de actores del sistema. Se puede hablar de una experiencia común a la totalidad de los miembros del sistema y de rutinas organizadoras que son compartidas también por el conjunto de éste. El mercado laboral se ve también reforzado por la existencia de una formación específica y conjunta con el actor por parte de las instituciones académicas de carácter público mayoritariamente y dirigidas a la calificación de los operarios, técnicos, enfermeros, médicos, doctores y directivos.

\subsubsection{LA GESTIÓN DE LA INNOVACIÓN}

El patrón de la gestión de la innovación que se produce en estos sistemas es también compartido y se puede definir como incremental y continuo. Es decir, a partir de una innovación determinada existe un proceso de mejoría continuada con una rápida y eficaz difusión entre los actores.

La difusión tecnológica más relevante es la informal. Esta difusión es consecuencia de la proximidad y familiaridad entre los miembros de los actores. Muchas son las actividades y los ámbitos que son compartidos por directivos y técnicos de los actores y que constituyen el vehículo de esta difusión informal.

También juegan un papel importante en la innovación y su difusión, las instituciones representativas. Dentro de estas entidades y de manera conjunta con los actores, se desarrollan proyectos innovadores que están al alcance del conjunto de los actores del sistema.

Otro aspecto importante de la difusión es la que se basa en las relaciones entre clientes y proveedores. El conocimiento mutuo, las relaciones de larga 
duración y la fuerte competencia entre los diferentes suministradores, incentiva la innovación y fomenta su difusión.

\subsubsection{LA RED COOPERATIVA}

Dentro del SI se producen un gran número de acuerdos de cooperación, tanto de carácter formal como informal. Esta cooperación se desarrolla en dos direcciones básicas. Por un lado, la cooperación horizontal que se establece entre los actores que operan en una misma fase productiva, podemos encontrar desde acuerdos simples de subcontratación de capacidad productiva hasta acuerdos más estables. La cooperación vertical se establece entre los suministradores de «inputs» y los fabricantes. Estas relaciones pueden calificarse de cooperativas en contraste con las relaciones competitivas frecuentes en otras formas organizativas.

Muchos de los acuerdos cooperativos nacen de una elección estratégica deliberada, como consecuencia de una respuesta conjunta frente a determinadas situaciones externas a las que se enfrentan los actores. El hecho destacable de estas relaciones cooperativas es su combinación con una fuerte rivalidad. La justificación a esta dualidad se puede encontrar en la consideración de la lucha entre competidores como un juego de suma no-cero. La mejoría de una empresa no va en detrimento de los competidores del sistema, la colaboración permite la mejoría del conjunto del sistema y puede traducirse en nuevos productos y nuevos mercados [Fernández y Conesa, 1996, pp. 61-147 y Becattini, 1972, p. 73].

\subsubsection{LA FLEXIBILIDAD}

La flexibilidad que tienen los actores del sistema nace como consecuencia de otra de sus características. La dimensión de los actores, las relaciones verticales y horizontales, les permiten rápidas adaptaciones a los cambios y la modificación de las características básicas de los procesos y productos.

La red de actores del SI puede adaptarse rápidamente y evitar impactos externos como los incrementos y decrementos de la capacidad productiva, el paso de una tecnología a la otra o los cambios en los mercados y en los productos.

Pero lo que es más importante es que la flexibilidad del SI puede considerarse como una flexibilidad o versatilidad activa relacionada con el aprovechamiento de las oportunidades de los mercados y no, una flexibilidad o adaptabilidad pasiva que constituiría una flexibilidad enfocada a la adaptación de los actores a las presiones externas del sistema [Sengenberger y Pyke, 1993, pp. 41—42]. 
En conclusión, estos recursos que hemos definido como compartidos por su carácter colectivo, tienen las características propias de los recursos generadores del éxito. Están basados en patrones de comportamiento y redes de relaciones entre los actores al nivel de sistema. Su carácter idiosincrásico consecuencia de un proceso histórico, hace que sean difícilmente reproducibles en otras localizaciones. El hecho de que en este desarrollo participe o no un actor individual sino grupos de actores de diversas actividades, dificulta aun más la apropiación de estos recursos por parte de los actores de fuera del sistema.

FIGURA 3.2. Recursos generadores del éxito del SI.

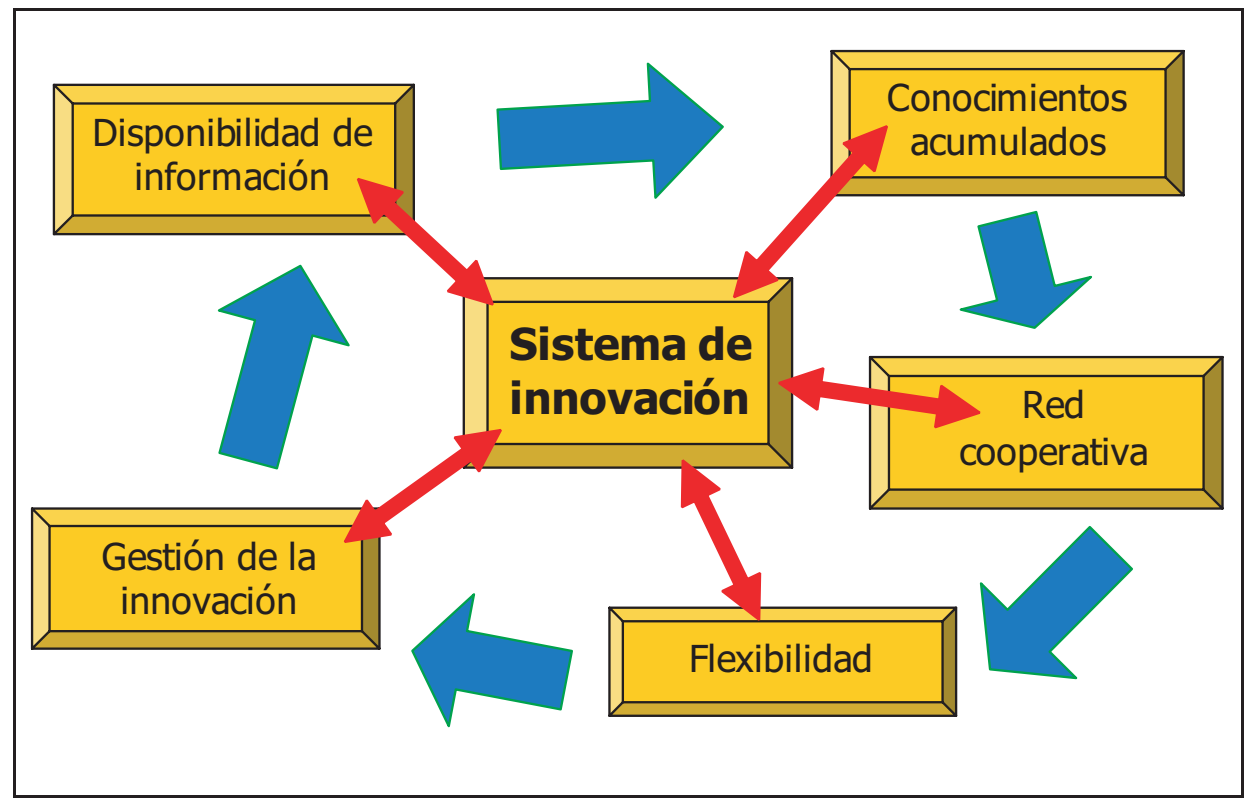

Fuente: Elaboración propia.

\subsection{HIPÓTESIS DEL ESTUDIO}

Las consecuencias del anterior desarrollo teórico sobre el éxito del SI se traducen en cuatro hipótesis básicas que están agrupadas en cuatro fases del análisis del estudio empírico del SSPC.

\section{El valor del presupuesto económico del actor del SSPC se relaciona} significativamente con los resultados de la aportación científica del actor al conjunto del SI-SSPC. 
Esta hipótesis refleja el hecho que el conjunto de recursos disponibles por el actor del SSPC puede estar relacionado directamente con su producción científica. Si partimos del punto de que el presupuesto del actor proviene del presupuesto del Estado, la comparación de los diferentes actores puede reflejar un desequilibrio en la distribución de éste, [esta hipótesis fue medida por el número de actividad científica, ensayos clínicos, publicaciones SCI y su factor de impacto, otras publicaciones, proyectos de investigación, tesis doctorales y trasplantes efectuados por los actores del SI-SSPC y definidas en el apartado 3.4.2.2 de este estudio. Esta hipótesis está contrastada en las fases 1,2,3 y 4 del estudio empírico —capítulo 7 de la tesis-].

\section{El número de personal de nivel superior que posee el actor del SSPC en plantilla se relaciona significativamente con los resultados de su aportación científica al conjunto del SI-SSPC.}

La segunda hipótesis manifiesta que no solo el presupuesto económico puede influir directamente en la producción científica del actor del SSPC. Una plantilla formada por personal de alta calificación puede permitir la producción de un mayor número de actividad científica con menor presupuesto [esta hipótesis fue medida con la producción por personal de nivel superior de las variables de la hipótesis anterior y está contrastada en las fases 1,2,3 y 4 del estudio empírico —capítulo 7 de la tesis-].

\section{La tradición histórica hospitalaria de Catalunya, la rivalidad y el alto grado de competencia que mantienen los hospitales del Grupo 4 se relaciona significativamente con su aportación científica al SI-SSPC.}

Lo que esta tercera hipótesis supone es que la historia hospitalaria de Catalunya tiene una fuerte relación con la estructura hospitalaria actual. Consecuentemente los resultados de esta estructura y la rivalidad que mantienen los hospitales del Grupo 4 son la fuente del éxito de estos actores [esta hipótesis fue medida con los índices de las variables comunes a los hospitales de alta tecnología del SI-SSPC y está contrastada en la fase 4 del estudio empírico - capítulo 7 de la tesis-].

\section{El papel que desempeñan los actores del SSPC, está de acuerdo con la misión y objetivos descritos en la LOSC y estos fomentan el desarrollo y la cooperación dentro del SI-SSPC.}


La curta hipótesis subraya que los actores del SI-SSPC tienen un papel decisivo en el éxito del mismo. La LOSC describe con todo el detalle la misión y objetivo de los actores del SSPC. Las empresas están desarrollando satisfactoriamente su misión dentro del sistema o tienen su capacidad limitada por la regulación existente, fomentan la dinamización y cooperación en el sistema o mantienen un grado de rivalidad y competitividad prejudicial para el mismo [esta hipótesis fue medida con la tabulación de las preguntas de la parte cualitatva de la encuesta y está contrastada en la fase 5 del estudio empírico —capítulo 7 de la tesis-].

\subsection{OBJETIVOS DEL ESTUDIO}

La revisión de la literatura sobre el SI nos servió para ver una serie de enfoques y teorías que han aportado, en mayor o menor medida, luz sobre los interrogantes centrales del SI. Este resumen nos ha permitido poder describir un marco teórico explicativo, dentro del enfoque conceptual de los SI. Por otro lado, el SI tiene sido objeto de estudio desde muy diversas perspectivas y diferentes niveles de análisis — de país hasta empresa—. Este conjunto heterogéneo de aportaciones a su estudio vino desde diferentes campos de conocimiento, con diferentes objetivos y metodologías y con aproximaciones a veces parciales y a veces globales de lo que significa esta realidad socioeconómica. La revisión de estas perspectivas, a pesar de su dispersión, nos ha dado una visión general del SI que nos permite una caracterización operativa del mismo. Para conectar esta caracterización con las causas del éxito hemos identificado la existencia de una serie de recursos compartidos por los actores que pertenecen al SI. Explicado esto, el objetivo genérico del trabajo es realizar un análisis de la forma organizativa del Sistema de Innovación en el Sector Sanitario Público Catalán a través del estudio de la estructura propuesta por la Llei d'Ordenació Sanitària de Catalunya.

Este objetivo genérico se concretó en los objetivos específicos que estructuran el trabajo y que son los siguientes:

(a) Realizar un inventario de los actores que desempeñan algún papel en el SI-SSPC.

(b) Establecer una tipología de los actores que permita definir su papel en el modelo SI-SSPC.

(c) Analizar para cada grupo de actores las actividades que realizan en el SI-SSPC. 
A partir de estos objetivos específicos (a), (b) y (c) se divide el estudio empírico en cinco fases diferenciadas — capítulo 7 de la tesis—que se definen a seguir:

1. Comparación interna o individualizada entre todos los actores del Sistema Sanitario Público Catalán. Para estudiar la producción y competitividad de ellos se compararon sus resultados cientificos, número de otras publicaciones, numero de personal de nivel superior y valor del presupuesto.

2. Comparación interna o individualizada entre las Regiones Sanitarias del Servei Català de la Salut. Para estudiar la aportación científica de las Regiones Sanitarias al SI-SSPC, se compararon sus resultados cientificos, número de otras publicaciones, número de personal de nivel superior y valor del presupuesto.

3. Comparación interna o individualizada entre las Empresas Públicas del Servei Català de la Salut. Para este estudio se analizó el papel que tienen estas empresas en el SI-SSPC, se compararon sus resultados cientificos, número de otras publicaciones, número de personal de nivel superior y valor del presupuesto.

4. Comparación interna o individualizada entre los hospitales de alta tecnología - Grupo 4- del SI-SSPC. Conjuntamente estos actores dominan la producción científica del SI-SSPC, además, mantienen un alto grado de rivalidad y competitividad. Para este estudio se analizó la producción de los hospitales comparando sus resultados de actividad cientifica, personal de nivel superior, presupuesto económico, número de camas, número de MIR —médico interno residente-, número de alumnos de medicina, número de publicaciones SCI, el factor de impacto de las publicaciones SCI - Science Citation Index-, número de otras publicaciones, número de tesis doctórales, número de ensayos clinicos, numero de proyectos de investigación y el número de trasplantes que realizan los hospitales de alta tecnología [estas variables están definidas en el apartado 3.4.2.27.

5. Análisis de los datos cualitativos de la encuesta hecha a los actores del SI-SSPC, estos datos corresponden a las preguntas de la parte 4 de la encuesta [este análisis está contrastado en la fase 5 del estudio empírico — capitulo 7 de la tesis—]. 
FIGURA 3.3. Objetivo de la tesis.

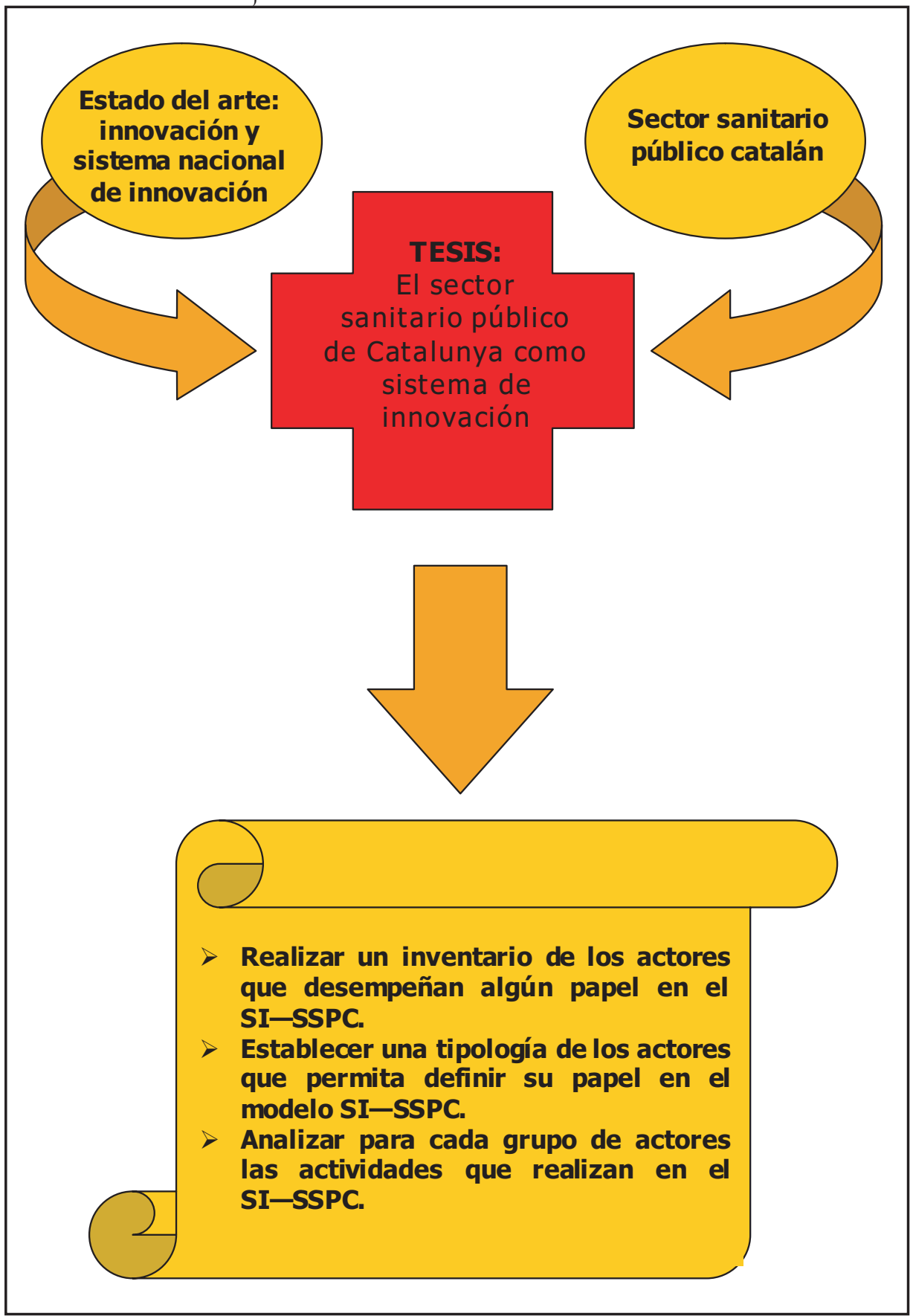

Fuente: Elaboración propia. 


\subsection{DISEÑO DEL ESTUDIO EMPÍRICO}

Una vez realizada la revisión teórica y la enumeración de las diferentes hipótesis, el trabajo empírico realizado se centró en los ámbitos de los actores del SSPC. El trabajo se organizó en dos partes. En la primera se hizo una aproximación teórica con el fin de caracterizarlos. En la segunda, se realizó el estudio empírico, para verificar las hipótesis formuladas.

\subsubsection{CARACTERIZACIÓN DE LOS ÁMBITOS DEL ESTUDIO EMPÍRICO}

Dentro de una primera aproximación al estudio de los ámbitos del presente trabajo realizó una introducción descriptiva para situar el tema y caracterizar los actores del sistema. Este análisis se estructuró de la siguiente manera:

1. Aproximación conceptual de salud, sanidad y medicina. También se ha realizado una aproximación conceptual de los sistemas sanitarios y de la demanda sanitaria.

2. Caracterización del sistema sanitario español a partir de su origen y desarrollo, la Ley General de Sanidad, el proceso de transferencias sanitarias a las Comunidades Autónomas por parte del INSALUD y el Informe Abril.

3. Caracterización del sector sanitario público catalán sus antecedentes históricos, la LOSC, el entorno económico, el mapa sanitario catalán, el plan de salud y el modelo sanitario catalán.

4. Caracterización de los actores del SSPC a partir del estudio de sus características, misión, objetivos, estructura funcional y demás aspectos propios de cada uno de ellos.

\subsubsection{ESTRUCTURACIÓN DEL TRABAJO EMPÍRICO}

El trabajo empírico de verificación de las hipótesis se realizó en cinco fases diferenciadas que se corresponden con los grupos de hipótesis formuladas.

1. Un análisis comparativo entre los actores del Sistema Sanitario Público Catalán.

2. Un análisis comparativo entre las regiones sanitarias del Servei Català de la Salut.

3. Un análisis comparativo de las empresas públicas del Servei Català de la Salut. 
4. Un análisis comparativo entre los hospitales de alta tecnología -Grupo 4- del SSPC.

5. Un análisis de los datos cualitativos de los actores del Sistema Sanitario Público Catalán.

Describiremos ahora cómo se preparó la confección de las muestras, explicaremos las fuentes de información, las medidas utilizadas, el periodo de tiempo analizado y las variables y las técnicas de análisis utilizadas.

En primer lugar analizaremos los rasgos comunes a todas las fases de la investigación y después especificaremos los rasgos particulares de cada fase.

\subsubsection{CONFECCIÓN DE LAS MUESTRAS Y FUENTES DE INFORMACIÓN}

El estudio empírico compara el comportamiento de las variables escogidas en diferentes ámbitos para un período de tiempo determinado. La causalidad de los resultados está implícita en el modelo teórico propuesto. Las comparaciones se hicieran sobre poblaciones de actores discriminados por su participación en un mismo entorno.

En el estudio empírico no se han determinado muestras representativas sino que se ha trabajado con la totalidad de las poblaciones de actores por tratarse de conjuntos relativamente pequeños.

Las fuentes de información fueron diversas y han sido especificadas para cada análisis en el apartado correspondiente. La descripción general es la siguiente:

Fuentes documentales primarias. Se utilizaron las memorias de actividad de los ejercicios, las cuentas de pérdida y ganancia, los balances e informes de gestión e información complementaria — estas fuentes están debidamente citadas en el capítulo de la bibliografía seleccionada-, también partiendo de los artículos y las series clínicas publicadas se capturaron exclusivamente publicaciones referenciadas en el «Medline» y «Current Contents», catalogadas como jounal article, firmadas por autores o instituciones integradas en los hospitales de alta tecnología y publicados en revistas indexadas con un factor de impacto superior a cero — Sistema del Factor de Impacto del Science Citation Index/Journal Citation Reports-, totalizando 2635 registros.

Fuentes documentales secundarias. Se utilizaron para algunos casos trabajos publicados por la Generalitat de Catalunya como la Enquesta de Salut de Catalunya 1994, el Pla de Salut de Catalunya 1993-1995 y el Pla de Salut de 
Catalunya 1996-1998 — estas fuentes están debidamente citadas en el capítulo de la bibliografía seleccionada-.

La Encuesta. El cuestionario utilizado para la obtención de los datos se incluye en el Anexo 3. Se estructura en cuatro partes: En la primera parte se recoge información de la persona responsable por la cumplimentación de la encuesta y en la segunda parte se transmite al actor entrevistado el resumen de nuestro estudio. En los apartados tercero y cuarto se pretende recoger los datos cuantitativos y cualitativos del actor. El tercero recoge los datos cuantitativos de la producción innovadora, de investigación y aportación científica del actor al SI-SSPC, también recoge los datos presupuestarios y de recursos humanos del actor. En el cuarto se solicita información sobre las relaciones con los otros actores del SI-SSPC, como también, los factores que influyen en la producción innovadora, de investigación y aportación científica del actor al SI-SSPC. Para la realización de la encuesta siempre se concertó una entrevista personal, lo que permitía la aclaración de posibles dudas.

Otras fuentes de información. Se utilizaron básicamente las entrevistas estructuradas y no estructuradas — todas ellas grabadas en cinta - realizadas a diferentes «expertos» en el tema y representantes de los actores objeto del estudio y que son las siguientes — la lista está confeccionada por orden alfabético del primer nombre de las personas entrevistadas-:

1) Albert Oriol i Bosch, Ph.D. Director d'Institut d'Estudis de la Salut.

2) Alfonso Ortiz. Hernández, Ph.D. Director General de Investigación Farmacéutica del Grup Ferrer.

3) Alicia Granados i Navarrete, Ph.D. Directora de l'Agència d'Avaluació de Tecnologia Mèdica.

4) Andrés Andrés Jara. Profesor del Departamento de Teoría Sociológica, Filosofía del Derecho y Metodología de las Ciencias Sociales de la Universitat de Barcelona.

5) Anna Roman Martinez: Cap de la Central de Balanços. Staff de Direcció de l'Àrea de Recursos Econòmics del Servei Català de la Salut.

6) Antoni Mateu i Serra. Gerent de la Regió Sanitària Lleida.

7) Antoni Trilla, Ph.D. Cap de Secció d'Epidemiologia Hospitalaria i Avaluació de Serveis Sanitaris. Direcció Tècnica d'Hospital Clínic de Barcelona.

8) Antonio Ramírez de Arellano Serna. Cap de l'Unitat de Gestió Econòmica d'Hospital General Vall d'Hebron. 
9) Antonio Ramírez de Arellano Serna. Profesor titular del Departamento de Teoría Económica de la Universitat de Barcelona.

10) Carles Casabayó i Queraltó. Gerent d'Institut de Diagnòstic per la Imatge.

11) Carlos Gutiérrez Pardo. Director Economicofinancero del Hospital Maternoinfantil Vall d'Hebron.

12) Carmen Martin Vega, Ph.D. Cap de Servei de l'Àrea de Recerca i Docència del Centre de Transfusió i Banc de Teixits.

13) Edi Madalena Fracasso, Ph.D. Catedrática de Administración de Empresas de la Universidad Federal de Río Grande del Sur — Brasil y Directora del Núcleo de Gestión de la Innovación Tecnológica NITEC.

14) Eduard Ribas i Palomera. Gabinet del Conseller de Sanitat i Seguretat Social de la Generalitat de Catalunya.

15) Emilia Sánchez i Ruiz: Cap de Pla de Salut i Avaluació. Àrea Sanitària del Servei Català de la Salut.

16) Esperança Marti $i$ Salís. Secretària del Patronat i Directora General de la Fundació Puigvert, IUNA.

17) Eva Torres Vives. Cap de Salut Laboral d'Ajuntament de Rubí.

18) Francesc Vives $i$ Vives. Gerent de la Regió Sanitària Tortosa.

19) Helena Ris Romeu. Gerent de la Regió Sanitària Centre.

20) Helena Segura i Badia. Secretari Executiu del Consell Català de la Formació Mèdica Continuada.

21) Isidre Parra Blázquez, Ph.D. Subdirector Gerent d'Hospital Universitari Germans Trias i Pujol.

22) Jaume Claramunt $i$ Baliellas. Cap de Programa de Coordinació d'Empreses d'Energètica d'Instalacions Sanitàries, S.A.

23) Jaume Tort i Bardolet. Cap del Gabinet de Direcció del Servei Català de la Salut.

24) Joan Grau i Sociats, Ph.D. Gerent de la Corporació Sanitària Clínic.

25) Joan Maria Adserà Gebellí. Gerent de la Regió Sanitària Tarragona.

26) Joan Maria Adserà Gebellí. Vicepresidente de Gestió i Prestació de Serveis de Salut.

27) Joan Rovira, Ph.D. Profesor titular del Departamento de Teoría Económica de la Universitat de Barcelona.

28) Joaquim Ramis i Coris, Ph.D. President de l'Acadèmia de Cièncias Mèdiques de Catalunya i de Balears.

29) Jordi Boix $i$ Roqueta. Gerent d'Institut d'Assistència Sanitària.

30) Josep Ganduxé i Soler, Ph.D. Gerent del Hospital Sant Joan de Déu. 
31) Josep Mañach i Serra. Gerent de la Regió Santària Barcelona Ciutat.

32) Josep Mañach i Serra. Gerent del Consorci Sanitari de Barcelona.

33) Josep Maria Borràs i Andrés, Ph.D. Director d'Institut Català d'Oncologia.

34) Josep Maria Padrosa i Macias. Gerent de la Regió Sanitària Barcelonès Nord i Maresme.

35) Josep Maria Padrosa i Macias. Gerent de la Regió Sanitària Girona.

36) Josep Maria Via $i$ Redons, Ph.D. Secretari del Govern de la Generalitat de Catalunya i de Relacions amb el Parlament.

37) Josep Ramon Casas Iglesias. Gerent de Gestió de Serveis Sanitaris.

38) Lluis Monset i Castells, Ph.D. Director General de Recursos Sanitaris del Departament de Sanitat i Seguretat Social.

39) Manuel Jovells Cases. Gerent d'Institut Català de la Salut.

40) María José Sendra i Cuesta. Cap de Control de Gestió d'Hospital General de Catalunya.

41) Meritxell Serra i San\%, Ph.D. Directora Economicofinancera de la Ciutat Sanitària i Universitària de Bellvitge.

42) Montserrat Betriu Monclús. Directora Economicofinancera d'Hospital General Vall d'Hebron.

43) Montserrat Grané i Alsina. Cap de la Unitat de Soport Operatiu de la Regió Sanitària Costa de Ponent.

44) Montserrat Gualdo Porredón. Directora Economicofinancera d'Hospital de Traumatologia i Reabilitació Vall d'Hebron.

45) Rafael Foguet Ambrós, Ph.D. Conseller Delegat del Grup Ferrer.

46) Ramon Sàrrias i Ramis, Ph.D. Vicesecretari del Col.legi Oficial de Metges de Barcelona.

47) Ricard Tresserras i Gaju, Ph.D. Cap del Servei d'Educació Sanitària i Programes de Salut de la Direcció General de Salut Pública.

48) Sueli Gonsalez Saes. Coordinadora Técnica de la Secretaria de Estado de la Salud del Estado de São Paulo - Brasil.

49) Susana Bigatà $i$ Viscasillas. Cap de Planificació i de Control de Gestió de Sistema d'Emergències Mèdiques S.A..

50) Victòria Petanás. Gabinet de Direcció del Servei Català de la Salut.

51) Xavier Bonfill i Cosp, Ph.D. Director del Servei d'Epidemiologia Clínica i Salut Pública d'Hospital de la Santa Creu i Sant Pau. 


\subsubsection{MEDIDAS UTILIZADAS Y PERIODOS DE TIEMPO ANALIZADOS}

Las medidas de resultados utilizadas son bianuales para ofrecer una indicación más exacta. El período de tiempo estudiado abarca los años 1996 y 1997. En el momento del inicio de este trabajo todavía no habían sido publicados los datos del año 1998. Asumiendo la conveniencia de estudiar períodos de tiempo largos, este estudio empírico precisó adaptarse a la disponibilidad de los datos que nos ofrecían las fuentes de información a nuestro alcance.

A pesar de esto, dos ejercicios representan, desde nuestro punto de vista, un equilibrio entre la necesidad de una perspectiva a largo plazo y la de reflejar las condiciones actuales de los actores.

Para la obtención de las medidas se agregaron resultados individuales de los actores durante el período de los dos años para obtener una media final de los valores usados en los cálculos.

Las medidas utilizadas en el estudio empírico de forma genérica han sido las siguientes:

Actividad científica. Se obtuvo a partir de la suma de actividad científica divulgadora, participación u organización de congresos nacionales e internacionales, simposios, cursos, jornadas, conferencias, actos, seminarios y otros [no se incluye en esta variable las publicaciones SCI u otras publicaciones que producen los actores del sistema y que son analizadas por separado y están definidas a seguir].

Alumnos de medicina. Se obtuvo a partir del número de alumnos de graduación de la facultad de medicina admitidos por los hospitales del Grupo 4.

Camas. Se obtuvo a partir del número de camas que poseen los hospitales del Grupo 4.

Ensayos clínicos. Se obtuvo a partir del número de ensayos clínicos realizados por los hospitales del Grupo 4.

Factor de impacto de las publicaciones SCI. Se obtuvo a partir del factor de impacto de las publicaciones del Science Citation Index-Journal Citation Reports de los hospitales del Grupo 4.

Médico interno residente - MIR - Se obtuvo a partir del número de MIR admitidos por los hospitales del Grupo 4.

Presupuesto económico. Se obtuvo a partir del presupuesto anual asignado a los actores del estudio.

Proyectos de investigación. Se obtuvo a partir del número de proyectos de investigación desarrollados por los hospitales del Grupo 4.

Publicaciones de artículos inéditos con factor de impacto - publicaciones $\underline{\mathrm{SCI}}$. Se obtuvo a partir del número de publicaciones SCI-JCR efectuadas 
por los hospitales del Grupo 4 [solamente se incluye en esta variable los artículos inéditos con factor de impacto del SCI-JCR].

Publicaciones de libros, capítulos de libros y demás publicaciones -otras publicaciones -. Se obtuvo a partir del número de publicaciones efectuadas por los actores del estudio [se incluye en esta variable todas las demás publicaciones producidas por los actores del SI-SSPC excluyendo las publicaciones SCI].

Personal de nivel superior. Se obtuvo a partir del total de profesionales de nivel superior de la plantilla de cada uno de los actores.

$\underline{\text { Tesis doctorales. }}$ Se obtuvo a partir del número de tesis leídas y aprobadas, dirigidas por miembros de los actores del sistema.

Trasplantes. Se obtuvo a partir del número total de trasplantes realizados por los hospitales del Grupo 4.

\subsubsection{TÉCNICAS UTILIZADAS EN EL ANÁLISIS DE LA ENCUESTA}

La información recogida a través de las fuentes ya descritas fue almacenada en soporte informático —-Microsoft ${ }^{\circledR}$ Access 97-, agrupada y revisada formando una base de datos con 945 registros.

El tratamiento dado a los datos contenidos en esta base general tuvo como objetivo:

La obtención de indicadores — tamaño y composición de la plantilla, cuantía de la financiación, etc. - quepermiten caracterizar el actor en los entornos del Sistema de Innovación y la determinación de la intensidad y el tipo de relaciones existentes entre los actores del SISSPC, con el fin de establecer su papel en el SI.

Para lograr el primer objetivo se emplearon técnicas simples de estadística descriptiva. En el caso de las variables continuas, se lograron medidas de centralización y de dispersión y para las variables discretas se calculó la distribución de frecuencias. Esto permitió determinar el perfil medio característico de cada uno de los tipos de actores objeto de análisis.

También se calculó el coeficiente de correlación de Pearson ${ }^{3}$ para un análisis bivariante

3 Coeficiente de correlación de Pearson: Medida de asociación lineal entre dos variables. Los valores del coeficiente de correlación varían entre -1 y 1 . El signo del coeficiente indica la dirección de la relación y el valor absoluto indica la intensidad. Los valores absolutos mayores indican que las relaciones son más fuertes. $R$ cuadrado: Medida de la bondad de ajuste de un modelo lineal. También recibe el nombre de coeficiente de determinación. Es la proporción de la variación de la variable dependiente explicada por el modelo de regresión. Sus valores van desde 0 a 1. Los valores pequeños indican que el modelo no se ajusta bien a los datos. $R$ cuadrado corregida: La $\mathrm{R}$ cuadrado muestral tiende a estimar de manera más precisa cuánto de bien se ajusta el modelo en la población. Habitualmente el modelo no se ajusta a la población tan bien como se ajusta a 
en las relaciones entre indicadores, con el que se pretendió determinar las relaciones entre los «inputs» con que cuenta el actor y las funciones que desempeña, los instrumentos que emplea, el tipo de actividad que más desarrolla y los resultados obtenidos.

Asimismo, también se recurrió al análisis factorial de correspondencias múltiples. Esta técnica de análisis multivariante resulta adecuada en aquellos casos en los que el número de variables es importante en relación con el número de observaciones y se sospecha la existencia de efectos de multicolinealidad entre las variables. En concreto, la técnica resulta especialmente útil cuando las variables a tratar son de carácter cualitativo. Una de las ventajas que se derivan de su aplicación es la reducción en la dimensión de los datos, ya que como resultado se obtiene un conjunto de ejes factoriales, ortogonales entre sí, que concentran toda la información contenida en el conjunto de variables. Esto permite establecer y analizar las relaciones entre ellas. La aplicación de esta técnica no permitió obtener conclusiones significativas, por lo que los resultados no fueron incluidos en el análisis.

El modelo utilizado está resumido en las tablas 3.1, 3.2, 3.3 y 3.4 que se muestran a seguir y donde son calculados los indicadores siguientes:

\section{Regresión Lineal}

Calculo de los indicadores:

- $\mathrm{R}$ - correlación de Pearson

- $\mathrm{R}^{2}$ - coeficiente de determinación

- $\mathrm{R}^{2}$ corregida

- Significación del cambio en F

TABLA 3.1. Grupo todos los actores.

\begin{tabular}{||l|l||}
\hline Variable dependiente & Variable independiente \\
\hline \hline Actividad científica & Personal de nivel superior \\
\cline { 2 - 2 } & Presupuesto \\
\hline \hline Otras publicaciones & Personal de nivel superior \\
\cline { 2 - 2 } & Presupuesto \\
\hline
\end{tabular}

Fuente: Elaboración propia.

la muestra de la que se ha derivado. La R cuadrado corregida intenta corregir la R cuadrado para reflejar más estrechamente la bondad de ajuste en la población. Significación del cambio en F: Nivel crítico para la hipótesis de que el cambio de R cuadrado es cero. 
TABLA 3.2. Grupo hospitales de alta tecnología.

\begin{tabular}{|c|c|}
\hline Variable dependiente & Variable independiente \\
\hline \multirow[t]{3}{*}{ Actividad científica } & MIR \\
\hline & \begin{tabular}{|l} 
Personal de nivel superior \\
\end{tabular} \\
\hline & Presupuesto \\
\hline \multirow[t]{3}{*}{ Camas } & MIR \\
\hline & Personal de nivel superior \\
\hline & Presupuesto \\
\hline \multirow[t]{3}{*}{ Ensayos clínicos } & $\overline{M I R}$ \\
\hline & Personal de nivel superior \\
\hline & Presupuesto \\
\hline \multirow[t]{3}{*}{ Otras publicaciones } & MIR \\
\hline & Personal de nivel superior \\
\hline & Presupuesto \\
\hline \multirow[t]{3}{*}{ Proyectos de investigación } & MIR \\
\hline & \begin{tabular}{|l} 
Personal de nivel superior \\
\end{tabular} \\
\hline & Presupuesto \\
\hline \multirow[t]{3}{*}{ Tesis doctorales } & $\overline{\mid M I R}$ \\
\hline & \begin{tabular}{|l} 
Personal de nivel superior \\
\end{tabular} \\
\hline & Presupuesto \\
\hline \multirow[t]{3}{*}{ Trasplantes } & MIR \\
\hline & Personal de nivel superior \\
\hline & Presupuesto \\
\hline \multirow[t]{3}{*}{ Publicaciones SCI } & MIR \\
\hline & Personal de nivel superior \\
\hline & Presupuesto \\
\hline \multirow{3}{*}{$\begin{array}{l}\text { Factor de impacto de las } \\
\text { publicaciones SCI }\end{array}$} & MIR \\
\hline & \begin{tabular}{|l|} 
Personal de nivel superior \\
\end{tabular} \\
\hline & Presupuesto \\
\hline \multirow[t]{3}{*}{ Número de alumnos de medicina } & MIR \\
\hline & Personal de nivel superior \\
\hline & \begin{tabular}{|l|} 
Presupuesto \\
\end{tabular} \\
\hline
\end{tabular}

Fuente: Elaboración Propia. 
TABLA 3.3. Grupo regiones sanitarias.

\begin{tabular}{|l|l||}
\hline Variable dependiente & Variable independiente \\
\hline \hline Actividad científica & Personal de nivel superior \\
\cline { 2 - 2 } & Presupuesto \\
\hline \hline Otras publicaciones & Personal de nivel superior \\
\cline { 2 - 2 } & Presupuesto \\
\hline
\end{tabular}

Fuente: Elaboración propia.

TABLA 3.4. Grupo empresas públicas.

\begin{tabular}{||l|l||}
\hline Variable dependiente & Variable independiente \\
\hline \hline Actividad científica & Personal de nivel superior \\
\cline { 2 - 2 } & Presupuesto \\
\hline \hline Otras publicaciones & Personal de nivel superior \\
\cline { 2 - 2 } & Presupuesto \\
\hline
\end{tabular}

Fuente: Elaboración propia.

Para la realización de todos los cálculos descritos hasta el momento se empleó el paquete estadístico SPSS ${ }^{\circledR}$ — Statistical Package for Social Sciences— versión 7.5.2.S para Windows ${ }^{\circledR}$. 
